\title{
CARACTERIZACIÓN DEL FITOPLANCTON DE LAS LAGUNAS DE ALTA MONTAÑA DE SIERRA NEVADA (GRANADA, ESPAÑA) EN RELACIÓN CON LAS CARACTERÍSTICAS FÍSICO-QUÍMICAS DEL MEDIO
}

\author{
P.M. Sánchez Castillo ${ }^{1}$, L. Cruz-Pizarro ${ }^{2}$ y P. Carrillo ${ }^{2}$ \\ 1. Departamento de Biología Vegetal, Facultad de Ciencias, Universidad de Granada. 18001 Granada. Spain. \\ 2. Departamento de Biología Animal, Ecología y Genética, Facultad de Ciencias, Universidad de Granada. 18001 \\ Granada. Spain.
}

Palabras clave: phytoplankton, high mountain lakes, Spain.

\begin{abstract}
CHARACTERIZATION OF THE PHYTOPLANKTON FROM HIGH MOUNTAIN LAKES OF SIERRA NEVADA (GRANADA, SPAIN) ACCORDING TO PHYSICAL AND CHEMICAL WATER CHARACTERISTICS

During the ice-free period of 1984, a comprehensive limnological survey was carried out on some small highmountain lakes in the Sierra Nevada. In this paper we present data concerning the structure and dynamics of their phytoplanktonic communities in relation to the changes in several physical and chemical parameters. Seasonal variations in the community succesion rate, chlorophyll-a values, and species diversity are also shown and discussed.
\end{abstract}

\section{INTRODUCCIÓN}

Los lagos de alta montaña, como los de latitudes extremas, se encuentran sometidos a condiciones climáticas rigurosas que, en buena medida, condicionan las características de sus aguas y por lo tanto la naturaleza de sus comunidades. Por su singularidad han sido objeto de un especial interés en limnología y son considerables los estudios que, desde muy diferentes aproximaciones se han llevado a cabo en ellos, particularmente en el centro y norte de Europa y en Norteamérica (Thomasson, 1956; Pennak, 1968; ANDERSON, 1970; ELLSWORTH, 1983; etc).

El conocimiento de tales ecosistemas en la Península Ibérica es mucho más restringido y durante mucho tiempo se ha limitado a los realizados en los Pirineos. Los trabajos de Margalef (1948), Carter (1970), CapblancQ (1972), GonLimnética, 5: 37-50 (1989)

(C) Asociación Española de Limnología, Madrid, Spain
ZÁlez Guerrero (1927), Margalef et al. (1975) y VILASECA (1978), incluyen información sobre el fitoplancton de dichos lagos, bien en estudios específicos o bien en otros más generales sobre su vegetación algal. Los trabajos que aportan datos sobre el fitoplancton de los lagos del resto de los sistemas montañosos son aún más puntuales (Margalef, 1949; González Guerrero, 1965; etc) y, concretamente, en el caso de Sierra Nevada, se reducen a los que Martínez (1975, 1977 , 1980, 1981) llevó a cabo en la laguna de La Caldera.

Los trabajos de CAPBlancQ \& LAville (1968), Margalef et al. (1975), Ferrari et al. (1975), Malueg et al. (1978) y Balvay \& Blavoux (1981), entre otros, permiten poner de manifiesto una serie de características comunes a este tipo de ecosistemas en relación con su composición fisico-química: aguas frías, saturadas de oxígeno, débilmente mineralizadas y de moderada o baja alcalinidad. No resulta fácil, sin embargo, a par- 
tir de los datos que ofrece la bibliografía sobre su fitoplancton, establecer una tipología característi$\mathrm{ca}$, aunque es frecuente la presencia de especies pertenecientes a los géneros Cyclotella, Tabellaria, Meridion, Mallomonas, Dinobryon, Peridinium y Ceratium, así como de numerosas desmidiáceas en aguas de pH ácido (BALVAY, 1978; FERraRi \& Villani, 1978; Margalef et al., 1975; SchANZ, 1984), mientras que cuando las asociaciones que se consideran incluyen especies ultraplanctónicas, suelen estar bien representados gran número de pequeños flagelados de los géneros Chromulina y Ochromonas, fundamentalmente (Pechlaner, 1967; CapblancQ, 1972; MartíNEZ, 1977).

En este trabajo, que es parte de un estudio extensivo global sobre la limnología de estos sistemas, se describe la estructura y evolución temporal de la comunidad fitoplanctónica de diez lagunas a lo largo del período libre de hielo. Se analizan asimismo las variaciones de algunos parámetros físicos y químicos de sus aguas.

\section{MATERIAL Y MÉTODOS}

\section{Área de estudio}

Las lagunas que se estudian están situadas entre los 2800 y los $3100 \mathrm{~m}$ de altitud y presentan un período libre de hielo que oscila entre los 5 y 6 meses. Se trata, por lo general, de cubetas someras y rodeadas de pastizales de alta montaña (borreguiles), a excepción de las lagunas de La
Caldera y Las Yeguas, más profundas (12 y $8 \mathrm{~m}$ de profundidad máxima, respectivamente) y desprovistas de vegetación litoral. La laguna de Las Yeguas se encuentra represada.

Morales et al. (1986) ofrecen detalles sobre la composición química de sus aguas, así como de algunas características morfométricas de las lagunas. Los aportes hídricos proceden del deshielo y la localización particular de cada laguna, junto al tamaño relativo de su área de captación, determinan la existencia o no de afluentes y efluentes superficiales. Las lagunas Virgen Superior, Aguas Verdes y Las Yeguas reciben agua, prácticamente durante todo el verano, a través de efluentes. Las dos primeras, además, son las únicas (de entre las estudiadas) con efluentes superficiales visibles, al comienzo de los cuales se presenta la vegetación reófila característica de tales ambientes (SÁNChEZ CASTILlO, 1984). La vegetación macrofítica, cuando existe, se restringe a franjas litorales, particularmente desarrolladas en las lagunas de Virgen Media, Aguas Verdes y Gemela. En la tabla 1 se presentan, resumidas, algunas de estas características.

\section{Muestreos y métodos de análisis}

El muestreo se llevó a cabo a lo largo del verano de 1984, con una periodicidad aproximadamente quincenal, en estaciones localizadas a una distancia de entre 3 y $5 \mathrm{~m}$ de la orilla, donde se tomaron muestras subsuperficiales.

Las medidas de temperatura y $\mathrm{pH}$ se tomaron «in situ», utilizando una sonda Hydrolab 4041.

Tabla 1.- Algunos parámetros limnológicos de las lagunas estudiadas Some limnological characteristics of the studied lakes.

\begin{tabular}{|c|c|c|c|c|c|c|c|c|c|c|}
\hline Lagunas & $\begin{array}{l}\text { Longitud } \\
\text { máxima } \\
\quad(m)\end{array}$ & $\begin{array}{c}\text { Anchura } \\
\text { máxima } \\
(m)\end{array}$ & $\begin{array}{l}\text { Profundidad } \\
\text { máxima } \\
(m)\end{array}$ & $\begin{array}{l}\text { Área } \\
\left(m^{2}\right)\end{array}$ & $\begin{array}{l}\text { Perimetro } \\
(m)\end{array}$ & $\begin{array}{l}\text { Vegetación } \\
\text { litoral }\end{array}$ & $\begin{array}{c}\text { Prados } \\
\text { inundados }\end{array}$ & $\begin{array}{c}\text { Efluentes } \\
\text { permanentes }\end{array}$ & $\begin{array}{c}\text { Efluentes } \\
\text { permanentes }\end{array}$ & $\begin{array}{c}U T M \\
\text { (30SVG) }\end{array}$ \\
\hline Yeguas & 90 & 40 & 8,0 & 2.530 & 215 & NO & SI & SI & $\mathrm{NO}$ & 661009 \\
\hline Virgen Superior & 50 & 12 & 1,5 & 246 & 108 & S1 & SI & SI & SI & 665008 \\
\hline Virgen Media & 20 & 10 & 0,5 & 154 & 49 & S1 & SI & NO & $\mathrm{NO}$ & 664009 \\
\hline Aguas Verdes & 50 & 30 & 2,5 & 1.315 & 136 & SI & SI & SI & SI & 674006 \\
\hline Río Seco Superior & 20 & 15 & 1.8 & 250 & 58 & S1 & NO & NO & NO & 692008 \\
\hline Río Seco & 80 & 30 & 2.0 & 1.920 & 185 & SI & SI & SI & NO & 694007 \\
\hline Río Seco Inferior & 20 & 15 & 0,6 & 240 & 56 & NO & SI & SI & NO & 696007 \\
\hline La Caldera & 190 & 140 & 12.0 & 20.700 & 536 & NO & NO & NO & NO & 708012 \\
\hline Gemela & 25 & 20 & 1.0 & 402 & 76 & SI & SI & SI & NO & 712008 \\
\hline Majano & 80 & 60 & 0.8 & 3.374 & 220 & SI & SI & SI & NO & 711007 \\
\hline
\end{tabular}


Para la determinación del oxígeno disuelto se siguió el método de Winkler y la alcalinidad se valoró volumétricamente (RoDIER, 1981). El fósforo reactivo soluble se determinó con el método de Murphy \& Riley (1962) y para los nitratos se utilizó el método de la Brucina (AMerican Public Health Association, 1965). En el caso de los silicatos, medidos como sílice reactiva, se siguió el método colorimétrico descrito en Golterman (1969). Para el estudio del fitoplancton, se filtró un volumen de 201 a través de un filtro de red de nytal de $10 \mu$ de diámetro de malla; el recuento se llevó a cabo en un microscopio invertido Leitz Labovert, siguiendo a UTERMOHL (1958), y se contaron, en cada caso, al menos 100 campos visuales a 400 aumentos. Para la medida de los pigmentos fotosintéticos se pasaron 21 de agua a través de un filtro de fibra de vidrio Whatman GF/C (5 cm de diámetro); la extracción se llevó a cabo con metanol al $100 \%$ de acuerdo con Holm-Hansen \& Riemann (1978), los extractos fueron medidos con un espectrofotómetro UV/Visible Perkin Elmer a $665 \mathrm{~nm}$, y los valores de concentración de clorofila se han obtenido siguiendo la expresión propuesta por TALling \& DrIVER (1963) para extractos metanólicos. Para la medida de la diversidad se ha utilizado el índice de Shannon (Shannon \& Weaver, 1963) y el de LEwIs (1978) para cuantificar la tasa de sucesión en la comunidad.

\section{RESULTADOS}

\section{Características del medio}

La tabla 2 resume los resultados obtenidos para los diferentes parámetros medidos, en las lagunas estudiadas.

\section{a) Temperatura}

El rango de temperatura observado es semejante al medido en aguas superficiales en los lagos de los Pirineos (Margalef et al., 1975) y, en general, corresponde al de lagunas someras y al de la zona litoral de lagos de mayor profundidad de alta montaña, e incluso del ártico, durante el verano (Thomasson, 1956). Las lagunas más someras (Río Seco, Río Seco Superior, Río Seco Inferior, Gemela y Majano), en las que el aporte hídrico cesa a comienzos del verano, registran los valores más elevados y presentan notables oscilaciones a lo largo del período libre de hielo. Por otra parte, aquellas lagunas de mayor volumen y/o que mantienen un aporte más continuado (La

Tabla 2.- Valores extremos, media y desviación típica de los parámetros fisico-químicos medidos.

Extreme values, mean values and standard desviation of the studied parameters.

\begin{tabular}{|c|c|c|c|c|c|c|c|}
\hline Lagunas & $\begin{array}{c}\text { Temperatura } \\
\left({ }^{\circ} \mathrm{C}\right)\end{array}$ & $p H$ & $\begin{array}{c}\mathrm{O}_{2} \\
(m g / l)\end{array}$ & $\begin{array}{c}\text { Alcalinidad } \\
(\text { meq } / l)\end{array}$ & $\begin{array}{l}\mathrm{PO}^{=} \\
(\mu g / l)\end{array}$ & $\begin{array}{l}\mathrm{NO}^{-} \\
(\mu g / l)\end{array}$ & $\begin{array}{c}\mathrm{SiO}_{2} \\
(m g / l)\end{array}$ \\
\hline \multirow[t]{2}{*}{ Aguas Verdes } & $5-14$ & $6-8,2$ & $7-9,1$ & $0,13-0,12$ & ND $-12,4$ & $\mathrm{ND}-70$ & $0,53-0,73$ \\
\hline & $9,7 \pm 3,9$ & $6,7 \pm 0,7$ & $8,2 \pm 0,8$ & $0,17 \pm 0,02$ & $3,54 \pm 6,1$ & $12 \pm 25,8$ & $0,59 \pm 0,1$ \\
\hline \multirow[t]{2}{*}{ Virgen Media } & $7-20$ & $6-6,7$ & $6,6-9,6$ & $0,14-0,26$ & ND $-12,4$ & $\mathrm{ND}-\quad 35$ & $0,53-0,81$ \\
\hline & $11 \pm 5,2$ & $6,3 \pm 0,3$ & $8,1 \pm 1,1$ & $0,21 \pm 0,04$ & $2,07 \pm 5,1$ & $12,6 \pm 13,3$ & $0,66 \pm 0,1$ \\
\hline \multirow[t]{2}{*}{ Virgen Superior } & $4-12$ & $6,2-7,0$ & $7,6-11,1$ & $0,35-0,61$ & ND - 18,6 & $\mathrm{ND}-63$ & $0,81-1,06$ \\
\hline & $7 \pm 2,8$ & $6,6 \pm 0,3$ & $8,7 \pm 1,3$ & $0,43 \pm 0,09$ & $3,1 \pm 7,6$ & $27,5 \pm 24,5$ & $0,93 \pm 0,1$ \\
\hline \multirow[t]{2}{*}{ Yeguas } & $6-17$ & $6,7-7,7$ & $6,7-9,3$ & $0,21-0,96$ & ND $-18,6$ & $\mathrm{ND}-42,0$ & $0,84-1,88$ \\
\hline & $11,2 \pm 4,2$ & $7,1 \pm 0,4$ & $7,8 \pm 1,0$ & $0,56 \pm 0,27$ & $3,1 \pm 7,6$ & $16,5 \pm 15,6$ & $1,34 \pm 0,4$ \\
\hline \multirow[t]{2}{*}{ Caldera } & $3-14$ & $6,4-7,1$ & $6,4-9,0$ & $0,28-0,42$ & ND $-12,4$ & $8,4-109,2$ & $0,22-0,39$ \\
\hline & $10,1 \pm 4,7$ & $6,8 \pm 0,3$ & $7,8 \pm 1,0$ & $0,31 \pm 0,05$ & $2,7 \pm 4,9$ & $50,8 \pm 38,2$ & $0,31 \pm 0,1$ \\
\hline \multirow[t]{2}{*}{ Gemela } & $1,5-21$ & $6,1-7,2$ & $6,7-9,2$ & $0,19-0,28$ & $7,1-28,8$ & $2,8-109,2$ & $0,48-1,0$ \\
\hline & $12,2 \pm 6,7$ & $6,6 \pm 0,4$ & $7,6 \pm 0,8$ & $0,22 \pm 0,02$ & $13,3 \pm 8,4$ & $37,8 \pm 36,1$ & $0,85 \pm 0,2$ \\
\hline \multirow[t]{2}{*}{ Majano } & $2-19$ & $6,7-7,9$ & $6,7-8,6$ & $0,29-0,65$ & $N D-43,4$ & $\mathrm{ND}-126,0$ & $0,98-1,76$ \\
\hline & $11,7 \pm 6,5$ & $7,4 \pm 0,5$ & $7,9 \pm 0,7$ & $0,39 \pm 0,12$ & $10,9 \pm 16,3$ & $24,6 \pm 46,6$ & $1,29 \pm 0,3$ \\
\hline \multirow{2}{*}{ Río Seco Superior } & $2,0-18$ & $6,2-6,6$ & $5,2-8,5$ & $0,15-0,19$ & ND - 18,6 & ND -126 & $0,14-0,92$ \\
\hline & $10,3 \pm 6,0$ & $6,4 \pm 0,1$ & $7,1 \pm 1,1$ & $0,17 \pm 0,01$ & $5,17 \pm 8,2$ & $31,0 \pm 47,4$ & $0,54 \pm 0,4$ \\
\hline \multirow[t]{2}{*}{ Río Seco } & $3,0-16,0$ & $6,1-8,4$ & $7,0-9,0$ & $0,06-0,10$ & $7,1-21,4$ & ND - 91 & $0.53-0.79$ \\
\hline & $11,1 \pm 5,4$ & $6,7 \pm 0,8$ & $7,5 \pm 0,7$ & $0,08 \pm 0,02$ & $13,7 \pm 4,4$ & $37,4 \pm 30,2$ & $0,66 \pm 0,1$ \\
\hline \multirow[t]{2}{*}{ Río Seco Inferior } & $11,0-20,0$ & $6,2-7,9$ & $6,8-7,6$ & $0,06-0,47$ & $10.5-49.6$ & $2,8-93,8$ & $0.31-1.34$ \\
\hline & $16,4 \pm 3,5$ & $6,9 \pm 0,6$ & $7,1 \pm 0,4$ & $0,16 \pm 0,17$ & $35,1 \pm 14,9$ & $36,1 \pm 34,1$ & $0,77 \pm 0,4$ \\
\hline
\end{tabular}


Caldera, Las Yeguas y Virgen Superior) presentan aguas más frías y mayor constancia térmica estacional.

\section{b) Oxígeno disuelto}

La concentración de oxígeno disuelto ha estado comprendida entre 5,2 y $11,1 \mathrm{mg} / \mathrm{l}$ (tabla 2), lo que supone, en la mayoría de los casos, niveles próximos a la saturación (media: 90-120). Su evolución estacional muestra un patrón que, en líneas generales, puede explicarse por diferencias de solubilidad en función de la temperatura: los valores más elevados coinciden con el final del período libre de hielo, mientras que las menores concentraciones se miden durante finales de agosto y principios de septiembre.

\section{c) Mineralización, pH y alcalinidad}

La naturaleza de la roca predominante sobre la que se asientan las lagunas (micasquistos) determina aguas poco mineralizadas, con valores de conductividad inferiores a $75 \mu \mathrm{S} / \mathrm{cm}$ (MORALES et al., 1986). El pH es ligeramente ácido o próximo a la neutralidad, aunque por su carácter poco

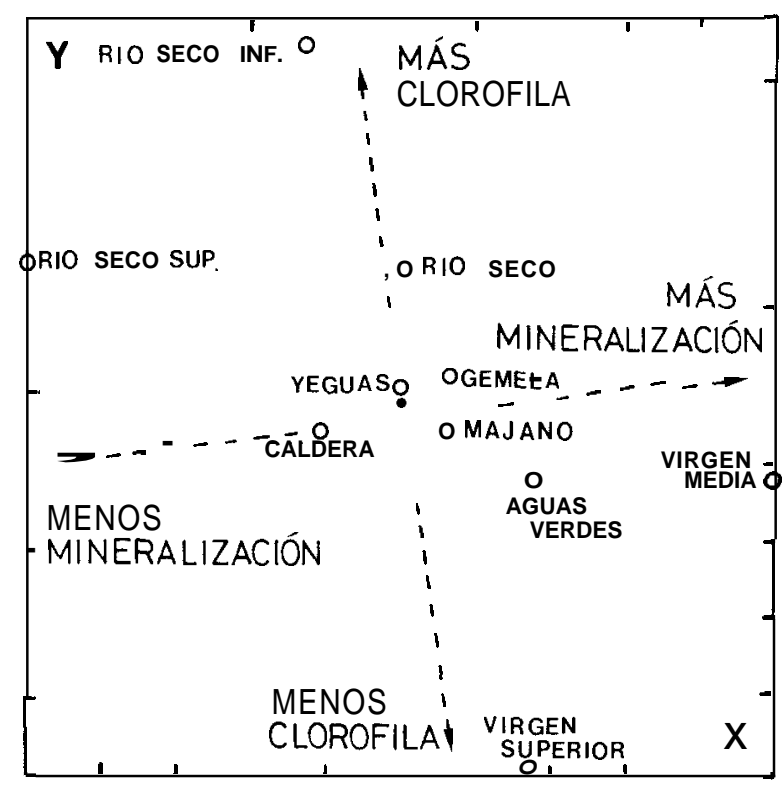

Figura 1.- Ordenación polar de las lagunas. Las líneas discontinuas indican la posición de sendos ejes de mineralización y concentración de clorofila.

Polar ordination of the lakes. The pair of dotted-lines axes represent the general direction of the mineralization and chlorophyll values. tamponado presenta en ocasiones bruscas oscilaciones; las lagunas de Majano y Las Yeguas muestran los valores medios más elevados $(7,4$ y 7,1 , respectivamente), mientras que en el resto estos valores son menores, y comprendidos entre 6,3 (Virgen Media) y 6,8 (La Caldera y Río Seco Inferior). En relación con la alcalinidad, es posible distinguir un grupo de lagunas (Aguas Verdes y las del valle de Río Seco) con valores medios inferiores a 0,2 meqll, y valores puntuales tan bajos como los medidos en Río Seco $(0,06 \mathrm{meq} / \mathrm{l})$ (tabla 2); en el resto, los valores medios han estado comprendidos entre 0,21 y 0,56 meqll.

\section{d) Fosfatos}

Un grupo de lagunas (Río Seco, Río Seco Inferior y Gemela) presenta, en todos los muestreos, valores superiores al límite de detección del método empleado, 0,03 $\mu \mathrm{g}$-at/l (tabla 2). Estas concentraciones, relativamente elevadas, se explicarían por el carácter más cerrado de estas lagunas, que determina una baja tasa de renovación de sus aguas; por otro lado, sus orillas se encuentran sometidas a una fuerte presión de pastoreo que puede producir enriquecimientos puntuales. La laguna de La Caldera (la de mayor volumen) y las de Las Yeguas, Aguas Verdes y Virgen Superior, de mayor tasa de renovación del agua, sólo presentan valores detectables de fosfato durante el período inmediatamente posterior al deshielo, de acuerdo con lo que observan PeCHLANER (1966); LARSON (1973) y FERRARI et al. (1975), entre otros, y que apoya los resultados de GroteRUD (1972) y BARICA \& ARMSTRONG (1971) que consideran la nieve como fuente principal de entrada de este nutriente, en ecosistemas similares. Las lagunas Virgen Media, Río Seco Superior y Majano no mostraron un patrón definido ni en la presencia de fósforo en el medio ni en relación con su evolución estacional.

\section{e) Nitratos}

Las concentraciones medias de nitratos han oscilado entre 12 y $\mathbf{5 0 , 8} \boldsymbol{\mu g} / \mathrm{l}$. Como en el caso de los fosfatos, no parece existir un único patrón de variación temporal, y se pueden distinguir tres tendencias. En las lagunas de Río Seco, Río Seco Inferior, Las Yeguas, Virgen Superior y La Caldera la concentración de nitratos se incrementa desde final del deshielo hasta mediados del perío- 
do de estudio (más o menos temprano a lo largo del mes de septiembre), cuando se inicia un descenso progresivo. En las lagunas de Majano, Gemela y Virgen Media la concentración de nitratos se incrementa notablemente hacia final del período de estudio; algo semejante observan FERRARI et al. (1975) en el lago Santo Parmense. Por último en las lagunas de Aguas Verdes y Río Seco Superior, los valores más elevados de la concentración de nitratos se miden a comienzos del período de estudio, por lo que se puede pensar que la principal fuente de entrada de nitrógeno al agua procede, como en el caso de los fosfatos, de la nieve.

\section{f) Silicatos}

La evolución estaciona1 de los silicatos sólo presenta marcadas fluctuaciones en aquellas lagunas en las que son abundantes las diatomeas. Este es el caso de la laguna de Las Yeguas, la única en la que el fitoplancton está dominado por este grupo (Synedra rumpens) durante más de la mitad del ciclo de estudio (fig. 4), y en la que se apre-

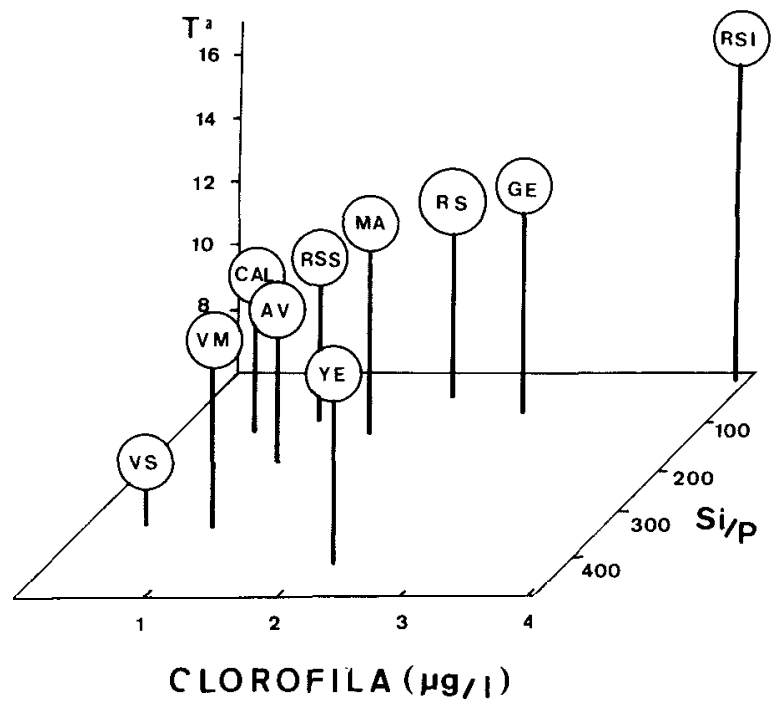

Figura 2.- Distribución de las lagunas en función de sus valores medios de temperatura $(" \mathrm{C})$, clorofila a $(\mu \mathrm{g} / \mathrm{l})$ y relación $\mathrm{Si} / \mathrm{P} . \quad \mathrm{VS}=$ Virgen Superior, $\mathrm{VM}=$ Virgen Media, $\mathrm{CAL}=$ Caldera, $\mathrm{AV}=$ Aguas Verdes, $\mathrm{RSS}=\mathrm{Río} \mathrm{Seco} \mathrm{Su}-$ perior, $\mathrm{YE}=$ Yeguas, $\mathrm{MA}=$ Majano, $\mathrm{RS}=$ Río Seco, $\mathrm{GE}=$ Gemela, RSI = Río Seco Inferior.

Distribution of lakes according to their temperature ("C), chlorophyll a $(\mu \mathrm{g} / \mathrm{l})$ and $\mathrm{Si} / \mathrm{P}$ ratio mean values. cia un marcado descenso en la concentración de silicatos, paralelo al máximo de abundancia de aquella especie. Por otro lado, el hecho de que en la laguna de La Caldera se haya medido la menor concentración de sílice reactiva, mientras que las de Majano y Río Seco Inferior, de entre las de menor volumen, presenten elevadas concentraciones, sugiere la influencia del volumen y de la profundidad en los procesos biológicos de reciclado de este nutriente (WETZEL, 1981).

Los trabajos de Tilman et al. (1982), Kilham \& Kilham (1984) y Tilman et al. (1984) ponen de manifiesto la importancia de la relación $\mathrm{Si} / \mathrm{P}$ en la determinación de la estructura de las comunidades fitoplanctónicas. Nuestros resultados confirman las agrupaciones que dichos autores establecen en función de los valores de esta relación; así, en Las Yeguas, la relación Si/P es de 533, con un fitoplancton dominado por diatomeas, mientras que en las lagunas de Río Seco Inferior, Río Seco y Gemela, donde las algas verdes constituyen el grupo fundamental, la relación alcanza sus mínimos valores, variando entre 24 y 71 .

\section{Estructura de las comunidades fitoplanctónicas}

En general, las diatomeas, clorofíceas y zigofíceas constituyen los grupos mayoritarios en la composición taxonómica del fitoplancton de las lagunas de Sierra Nevada. La presencia y distribución de las distintas especies en el conjunto de las lagunas se muestra en Sánchez Castillo (1988), y en la tabla 3 se indican las especies más frecuentes en cada laguna. Tan sólo Cymbella minuta y Nostoc kihlmani aparecen de forma general en las lagunas estudiadas, coincidiendo la aparición de esta última con situaciones críticas en la concentración de nitrato.

El análisis de ordenación polar (Bray \& Curtis in $\mathrm{GAUCH}_{\mathrm{A}}$ 1986) se ha realizado a partir de los valores de similitud entre las lagunas, calculados sobre las abundancias específicas. Esto permite ordenar las lagunas en grupos más o menos definidos (fig. 1) que, en buena medida, parecen explicarse por su posición sobre sendos ejes de mineralización y clorofila, temperatura y relación Si/P (fig. 2).

Las lagunas del grupo constituido por Virgen Superior, Virgen Media y Aguas Verdes mues- 
tran en común algunos táxones de crisofíceas, diatomeas y zigofíceas, particularmente Chromulina nevadensis (SNChez Castillo, 1987a), Fragilaria pinnata, Tabellaria flocculosa y Cosmarium laeve. Las lagunas de Virgen Superior y Virgen Media comparten además Cosmarium novae-semliae, Staurastrum hirsutum y Scenedesmus pseudohystrix. Este grupo de lagunas presenta bajas concentraciones de clorofila (inferiores a $1 \mu \mathrm{g} / \mathrm{l}$ ), va-

Tabla 3.- Distribución de las especies de presencia más continuada en cada laguna. 1, Yeguas; 2, Virgen Superior; 3, Virgen Media; 4, Aguas Verdes; 5, Río Seco superior; 6, Río Seco; 7, Río Seco Inferior; 8, Caldera; 9, Gemela; 10, Majano.

Distribution of the most common species in the lakes.

\begin{tabular}{|c|c|c|c|c|c|c|c|c|c|c|}
\hline \multirow[b]{2}{*}{ Especies } & \multicolumn{10}{|c|}{ Localidades } \\
\hline & 1 & 2 & 3 & 4 & 5 & 67 & 78 & 9 & 1 & 0 \\
\hline Synechococcus maior & & + & + & & & + & + & & + & + \\
\hline Cyanarcus sp. & & & & & & & & + & & \\
\hline Nostoc kihlmani & + & + & + & + & + & + & + & & + & \\
\hline Oscillatoria agardhii & & + & & + & & & & & & \\
\hline Oscillatoria planctonica & + & & & & + & + & + & + & & \\
\hline Chromulina mikroplankton & & & & + & + & + & & + & & \\
\hline Chromulina nevadensis & & + & + & + & & & & & & \\
\hline Chromulina rosanoffi & & & & & & & & + & & \\
\hline Cyclotellasp. & & & & & + & & & & & \\
\hline Diatoma hiemale mesodon & + & + & & + & & & & + & + & \\
\hline Fragilaria pinnata pinnata & & + & + & + & & & & + & & \\
\hline Synedra rumpens & + & + & & + & + & + & + & & + & + \\
\hline Tabellaria flocculosa & & + & + & + & & & & & & \\
\hline Achnanthes minutissima & & + & & + & & & & + & + & \\
\hline Pinnularia viridis & + & & & + & & + & & & + & + \\
\hline Cymbella minuta & + & + & + & + & & + & + & + & + & + \\
\hline Nitzschia hantzschiana & + & & & + & + & & + & + & + & \\
\hline Surirella tenera & + & & & & & + & + & & & \\
\hline Euglena spirogyra & & & & & + & & + & & & \\
\hline Chlamydomonus cf. coniformis & & & & & & + & & & & \\
\hline Chlamydomonas sp. & + & & & & & & & & + & \\
\hline Chlorangiella pygmaeu & & & & & & & & & + & \\
\hline Ankistrodesmus falcatus & & & & & & & & & + & + \\
\hline Korshikoviella gracilipes & & & & & + & + & + & & & \\
\hline Oocystis lacustris & & & & & + & + & & + & + & \\
\hline Pediastrum boryanum & & + & + & + & & + & & & + & + \\
\hline Scenedesmus pseudohysthrix & & & + & + & & & & & + & + \\
\hline Scenedesmus quadrispina & & & + & & & & & + & + & + \\
\hline Cylindrocystis brebissonii & & & & & & & + & & & \\
\hline Actinotaenium globosum & & & + & + & & + & & & + & \\
\hline Cosmarium laeve & & + & + & + & & + & + & & & \\
\hline Cosmarium novae-semliae & & & + & + & & & & & & \\
\hline Cosrnarium regnelli & & & + & & & + & & & & \\
\hline Staurastrum dilatatum & & & & + & & & & & & \\
\hline Staurastrum hirsutum & & & + & + & & & & & + & + \\
\hline Staurastrum punctulatum & & & & & & & & & + & + \\
\hline
\end{tabular}

lores relativamente bajos de temperatura y una relación Si/P elevada (192-336); el fósforo muestra valores medios comprendidos entre 2 y 3,5 $\mu \mathrm{g} / \mathrm{l}$. La laguna de Virgen Superior que, junto con la de La Caldera muestra las mayores concentraciones de nitrato, presenta importantes poblaciones de cianofíceas, mientras que en las lagunas de Virgen Media y Aguas Verdes predominan las zigofíceas y diatomeas. Estas últimas lagunas se podrían incluir en el grupo de lagos oligotróficos de desmidiáceas y diatomeas de Hutchinson (1967). En otras lagunas, los pequeños flagelados del género Chromulina son los táxones mejor representados Pechlaner (1967) y CAPBlancQ (1972), entre otros, ponen de manifiesto la importancia de estas pequeñas crisofíceas en el fitoplancton de este tipo de lagos.

Otro grupo diferenciable es el constituido por tres lagunas del valle de Río Seco: Río Seco Superior, Río Seco y Río Seco Inferior (fig. 1). Se caracteriza por presentar valores relativamente altos de temperatura media y baja alcalinidad (medias de 0,09-0,17 meq/l), aunque son muy diferentes en cuanto a la concentración de fosfatos y de clorofila (fig. 9). Su fitoplancton está dominado fundamentalmente por diferentes fases de Korshikoviella gracilipes, especie dotada de gran capacidad colonizadora.

El grupo constituido por las lagunas Gemela y Majano presenta un fitoplancton dominado por clorofíceas (fig. 5), entre las que los géneros $\mathrm{Pe}$ diastrum, Oocystis y Scenedesmus son los mejor representados. Aunque se trata de comunidades semejantes a las descritas por HuTCHInson (1967) como fitoplancton eutrófico de clorococales, también muestran similitud con el fitoplancton oligotrófico de Oocystis. Puede considerarse la laguna Gemela más eutrófica que la de Majano. Ambas presentan los mayores valores de temperatura media, la concentración de fosfatos relativamente elevada (en promedio 13,3 y $10,9 \mu \mathrm{g} / \mathrm{l}$, respectivamente), y valores de clorofila intermedios $(2,5$ y $1,5 \mu \mathrm{g} / \mathrm{l}$ ) (fig. 9).

Por último, el fitoplancton de las lagunas de $\mathrm{La}$ Caldera y Las Yeguas es, hasta cierto punto, diferenciable del resto de los grupos, por lo que no se han adscrito en la ordenación a ninguno de los establecidos. La Caldera, debido al desarrollo e importancia de las especies de crisofíceas, así como a los bajos valores de clorofila y tempera- 
tura, está más próxima al primer grupo de los descritos, mientras que la laguna de Las Yeguas, que presenta los valores más altos de la relación $\mathrm{Si} / \mathrm{P}$, muestra un fitoplancton dominado por diatomeas.

a) Dinámica estacional

Se ha analizado con detalle la variación estacional del fitoplancton en cinco de las lagunas estudiadas, que representan a cada uno de los grupos anteriormente descritos, aunque se ponen de manifiesto, además, las particularidades del resto de las lagunas, en cada grupo. La dominancia de diatomeas, tanto inmediatamente después del deshielo como al final del verano, cuando las temperaturas vuelven a descender, suele ser una característica general en el conjunto de las lagunas. Observaciones semejantes han sido realizadas, entre otros, por REYNOLDS (1984), Round (1981) y Sommer (1981).

\section{Laguna de Aguas Verdes}

A lo largo del período de estudio, el fitoplancton de esta laguna se caracteriza fundamentalmente por el predominio de las diatomeas, y por las elevadas tasas de sucesión entre sus poblaciones. Las especies de presencia más continuada son Diatoma hiemale var. mesodon, Tabellaria flocculosa, Achnanthes minutissima, Cymbella minuta y Nitzschia hantzschiana, entre las diatomeas; y Cosmarium laeve y Staurastrum dilatatum, entre las zigofíceas. Después del deshielo se miden los valores más elevados del índice de sucesión (0,109 unid. suc./día) al aparecer un numeroso grupo de especies con escasa representación numérica, cuya presencia no se mantiene en los siguientes muestreos; destacan, en esta situación, las zigofíceas (fig. 3), entre las que se encuentran, además de las especies antes mencionadas, Cosmarium novae-semliae y Staurastrum hirsutum. Entre finales de julio y principios de agosto se detectan las máximas concentraciones de nutrientes, tanto de fosfatos como de nitratos (los silicatos, por el contrario, presentan concentraciones más uniformes a lo largo del período de estudio). Hacia la mitad de dicho período (septiembre), se produce el único cambio cualitativo importante en el fitoplancton, debido a la aparición de dos especies de crisofíceas: Chromulina nevadensis y Chromulina mikroplankton; en este mo- mento, la concentración de nutrientes es muy baja, y sólo cabe destacar un notable descenso de la temperatura y un incremento en la concentración de oxígeno. Al final del período de estudio, las poblaciones de diatomeas vuelven a constituir una importante fracción de la comunidad.

En las lagunas Virgen Superior y Virgen Media se observa una evolución estacional semejante. Destaca la importancia de las desmidiáceas (Actinotaenium globosum y Cosmarium regnelli)
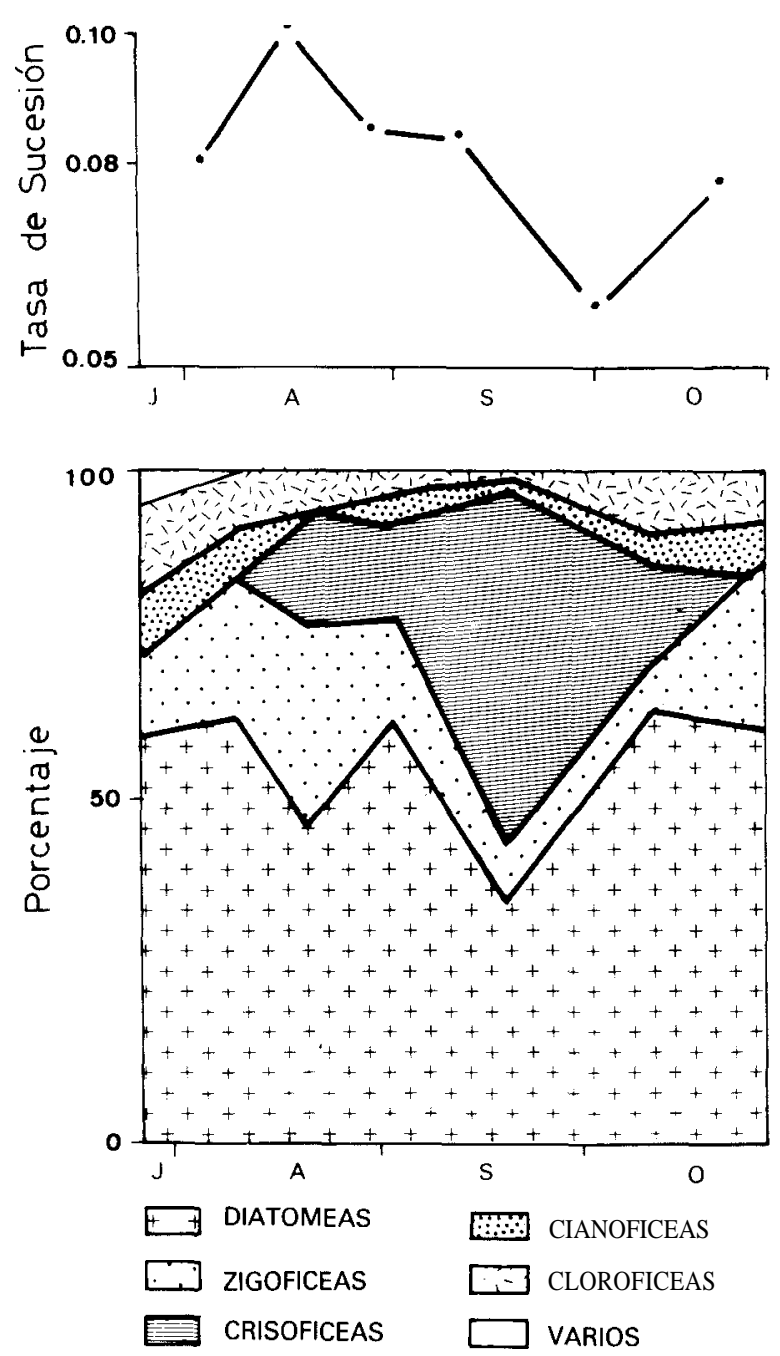

Figura 3.- Variación en la estructura de la comunidad y tasa de sucesión (unidadesldía) en la laguna de Aguas Verdes. Variation on the comrnunity structure and succession rate (units/day) in the lake Aguas Verdes. 
en Virgen Media, mientras que en Virgen Superior este grupo apenas está representado, las crisofíceas son menos importantes en su fitoplancton, y aparece una notable población de la cianofícea Oscillatoria agardhii.

Como ya se apuntó, estas tres lagunas se caracterizan por la naturaleza efímera de sus poblaciones, ya que, a excepción de las diatomeas (la mayoría de origen bentónico), el resto presentan una permanencia muy limitada en el tiempo. Creemos que la existencia de afluentes y efluentes, que dan lugar a una mayor tasa de renovación del agua en estas lagunas, contribuiría a explicar los altos valores que hemos medido en la tasa de sucesión de sus poblaciones.

\section{Laguna de Las Yeguas}

En la dinámica estacional del fitoplancton de esta laguna es posible diferenciar dos períodos (fig. 4). En el primero, desde el deshielo hasta finales de agosto, la comunidad está dominada por Chlamydomonas sp., y es la época en que se detectaron fosfatos y se midieron las concentraciones de nitratos mínimas, posiblemente debido al consumo de dicha población, que muestra una gran actividad biológica, como se pone de manifiesto por la presencia de numerosos individuos en fase de producción de zoosporas; en muestreos sucesivos, la presencia de Nostoc kihlmani coincide con un incremento en la concentración de nitratos. En el segundo, hacia la mitad del período libre de hielo, la comunidad pasa a estar dominada por diatomeas (con Synedra rumpens como especie más abundante) y paralelamente se detecta un descenso en la concentración de silicatos, que tan sólo a final del verano, cuando disminuyen las densidades poblacionales, se incrementa progresivamente. El índice de sucesión (fig. 4) alcanza sus valores máximos en los momentos de transición entre ambas situaciones.

\section{Laguna Gemela}

Las lagunas de este grupo (Gemela y Majano) presentan un fitoplancton dominado por clorofíceas (fig. 5). El índice de sucesión presenta los mayores valores a comienzos del período de estudio, debido a la alta tasa de renovación de las poblaciones como consecuencia de la presencia en el medio de especies ticoplanctónicas. La fase de mayor estabilidad en la estructura de la comuni-
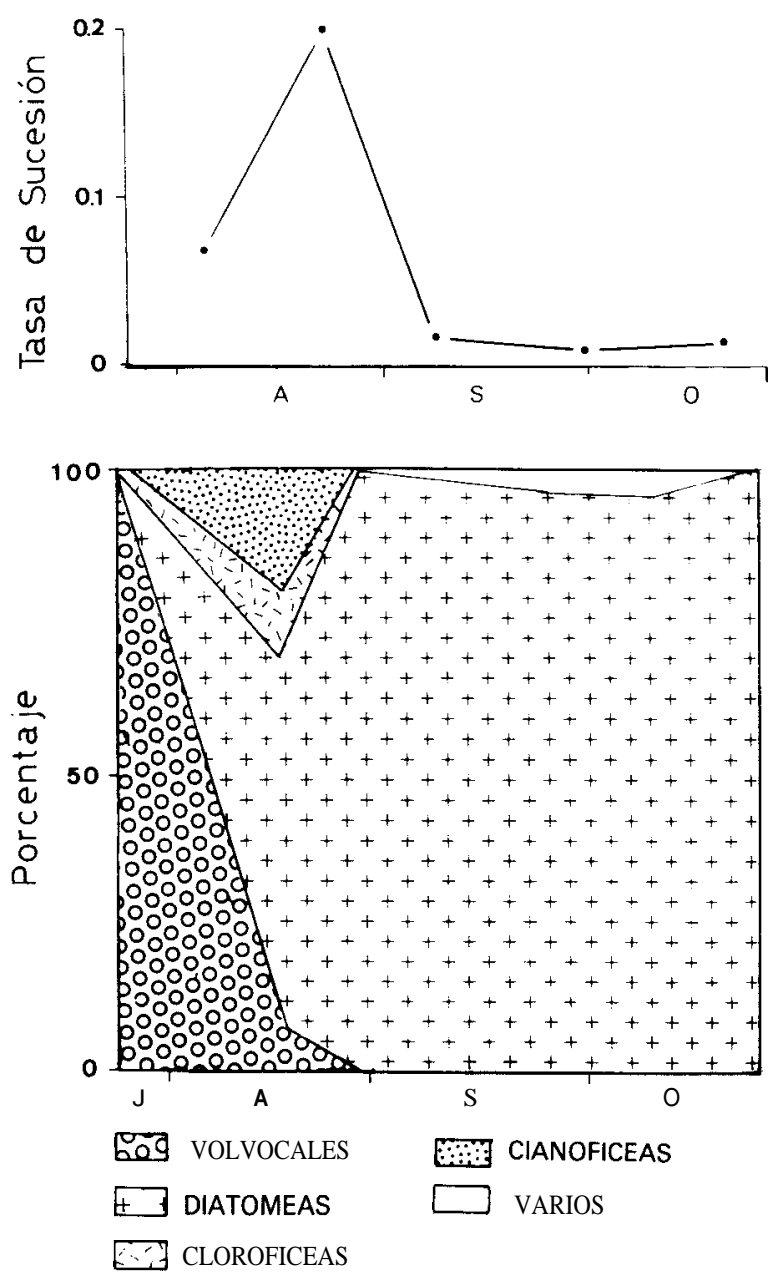

Figura 4.- Variación en la estructura de la comunidad y tasa de sucesión (unidades/día) en la laguna de Las Yeguas. Variation on the cornmunity structure and succession rate (units/day) in the lake Las Yeguas.

dad se inicia a mediados de agosto, con la desaparición de la mayoría de las especies planctónicas accidentales y la presencia de Chlorangiella pygmaea y Ankistrodesmus falcatus. La aparición y el desarrollo de Oocystis lacustris, Scenedesmus pseudohystrix y Pediastrum boryanum var. cornutum coincide con la desaparición de $A$. falcatus y Ch. pygmaea, hacia finales del período libre de hielo. Tanto en la laguna Gemela como en la de Majano, coincidiendo con un brusco descenso de la temperatura durante los últimos muestreos, 

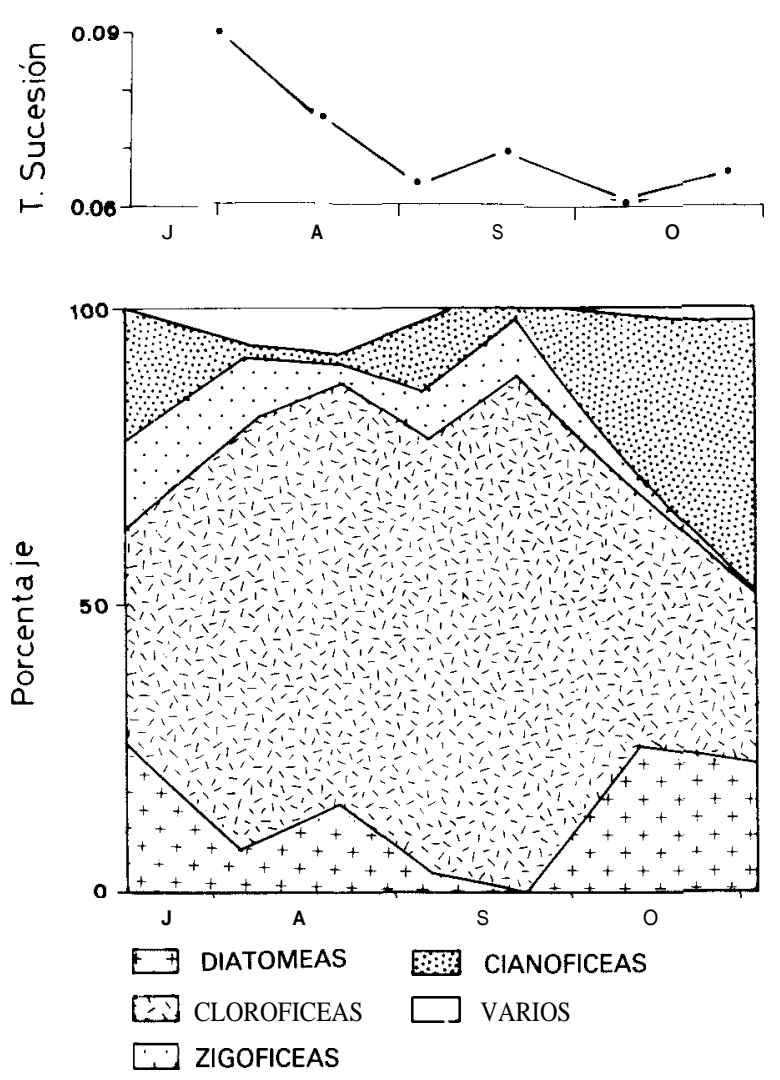

Figura 5.- Variación en la estructura de la comunidad y tasa de sucesión (unidadesldía) en la laguna Gemela.

Variation on the community structure and succession rate (unitslday) in the lake Gemela.

aparece una importante población de Synechococcus maior.

\section{Laguna de La Caldera}

Esta laguna es una de las de mayor volumen $\left(107.600 \mathbf{~ m}^{\mathbf{3}}\right)$ y profundidad (12 m) de Sierra Nevada y ha sido la más estudiada. MARTínez (1977, 1980, etc) estudia su fitoplancton y su evolución espacio-temporal en la zona limnética; algunos de los táxones que cita, como Rhodomonas minuta var. nannoplanctonica no han sido encontrados en la zona litoral (al igual que otros pequeños flagelados, puede haber escapado al tipo de muestreo realizado).

A comienzos del príodo jibre de hielo, la comunidad está constituida por crisofíceas y diatomeas en proporciones similares, aunque más de la mitad de las primeras son formas enquistadas.

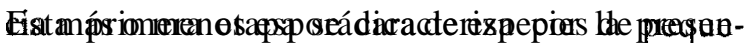
no tamaño (Achnanthes minutissima, Diatoma hiemale var. mesodon y Nitzschia hantzschiana. entre otras), que se mantienen hasta mediados de agosto, cuando la comunidad está dominada por Oocystis lacustris (que alcanza su máximo de-
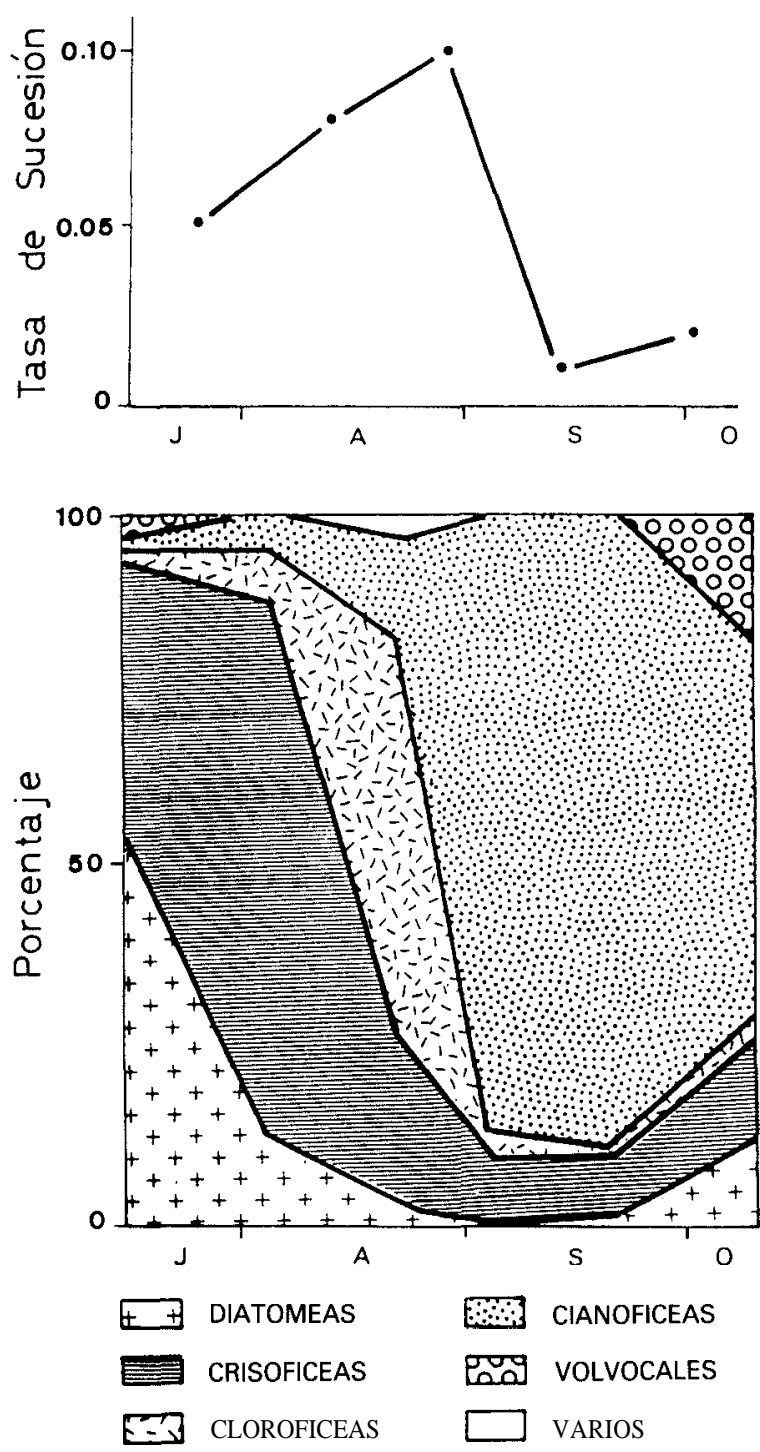

Figura 6.- Variación en la estructura de la comunidad y tasa de sucesión (unidadesldía) en la laguna de La Caldera.

Variation on the community structure and succession rate (units/day) in the lake La Caldera. 
sarrollo poblacional) y Oscillatoria planctonica, y aparecen por primera vez individuos de Cyanarcus sp. Las crisofíceas están representadas por Chromulina mikroplankton fundamentalmente, frecuente en otras regiones montañosas como los Alpes (Messikommer, 1942; Pechlaner, 1967).

Entre finales de agosto y principios de septiembre cambia de nuevo la estructura de la comunidad (fig. 6) y se miden los valores más altos de la tasa de sucesión, debido, principalmente, a la disminución de la concentración de $\boldsymbol{O}$. lacustris y al incremento del tamaño poblacional de Cyanarcus sp., que coincide con la máxima concentración de nitratos. Algo semejante observó Martínez $(1975,1977)$ en la zona limnética, lo que parece sugerir, como apunta TuRNOWSKY (1961) que esta cianofícea aprovecha las altas concentraciones de este nutriente para su desarrollo.

\section{Laguna de Río Seco}

Las lagunas del valle de Río Seco presentan un fitoplancton constituido fundamentalmente por distintas fases de Korshikoviella gracilipes (SÁNChez Castillo, 1987b), siendo en la laguna de Río Seco donde se desarrolla una población más estable. Las diferencias más notables observadas entre las tres lagunas se refieren a la naturaleza de las poblaciones que se establecen al comienzo y al final del período libre de hielo. Río Seco Inferior presenta, a comienzos del ciclo, una importante población de Cylindrocystis brebissonii; posteriormente $K$. gracilipes se hace dominante y, al final del ciclo, ésta es sustituida por Euglena spirogyra. En la laguna de Río Seco Superior el final del ciclo se caracteriza por la existencia de diatomeas en elevada proporción.

El desarrollo inicial del fitoplancton en la laguna de Río Seco, muestra una tasa de sucesión muy elevada (fig. 7). La existencia de un gran aporte hídrico procedente del deshielo, condiciona que a las especies típicamente planctónicas, tales como Chlamydomonas cf. coniformis y Chromulina mikroplankton, se les unan otras de carácter ticoplanctónico procedentes de los prados inundados circundantes, tales como Pinnularia viridis y Surirella tenera, entre otras. A principios de agosto cesa este aporte, por lo que se reduce considerablemente la riqueza específica y aumenta el índice de sucesión (fig. 7). A lo largo de este mes, el número de especies presentes no varía sustan- cialmente, si bien se produce una substitución de especies que, junto con el desarrollo en masa de $K$. gracilipes, determinan los valores más elevados del ciclo en cuanto a la tasa de sucesión (fig. 7). Podemos considerar que con la aparición de K. gracilipes en su estadio clorangioide se inicia
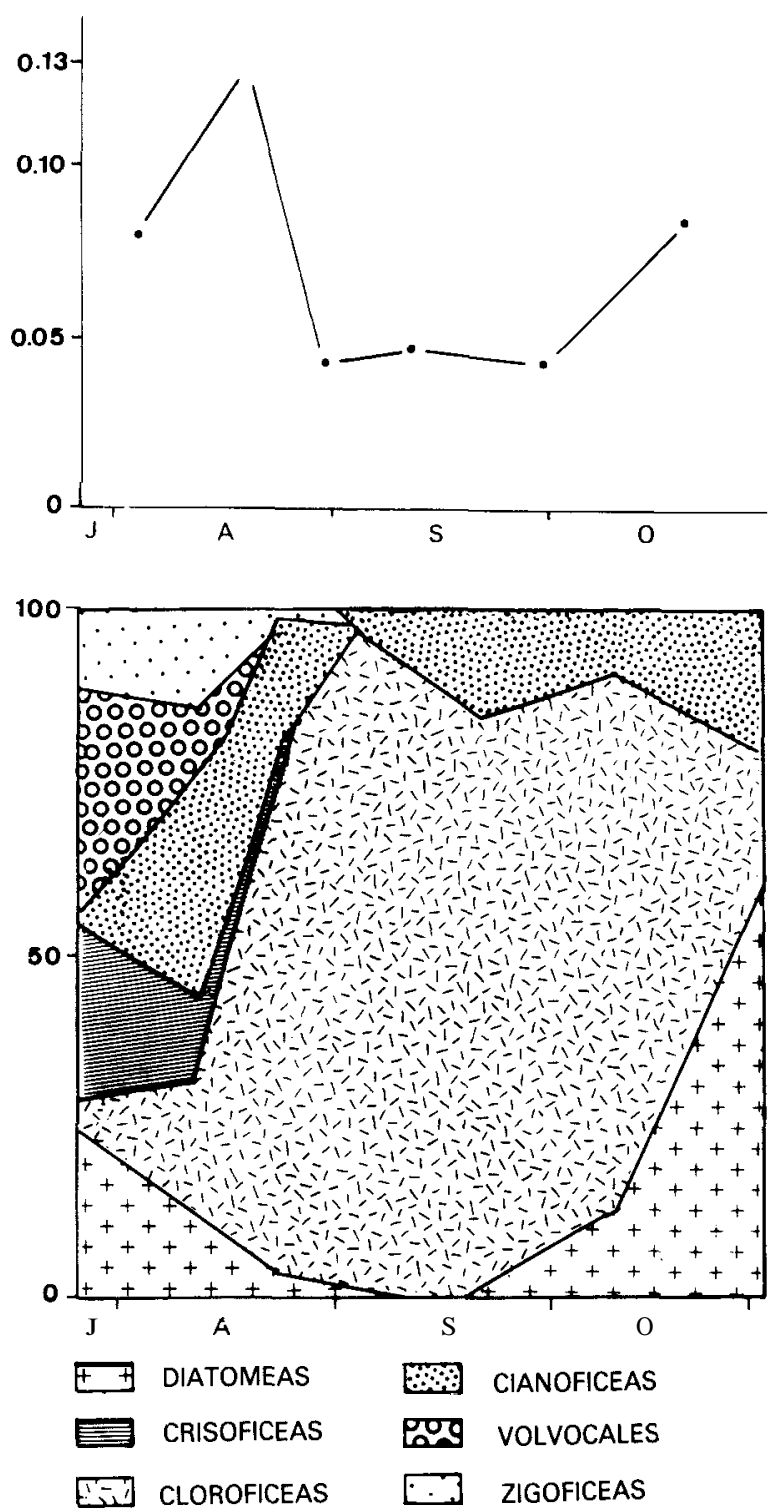

Figura 7.- Variación en la estructura de la comunidad y tasa de sucesión (unidades/día) en la laguna de Río Seco. Variation on the community structure and succession rate (unitslday) in the lake Río Seco. 
la fase más estable en la estructura de la comunidad, cuando predominan las clorofíceas. Es de destacar la evolución de $K$. gracilipes durante este período, observándose la mayor densidad de individuos en la fase ankiroide, a principios de septiembre.

\section{b) Diversidad específica}

El índice de diversidad, en el conjunto de las lagunas, varía entre 2 y 4 bitslindividuo. Estos valores se mantienen más o menos uniformes a lo largo del ciclo de estudio y, en todo caso, las va-

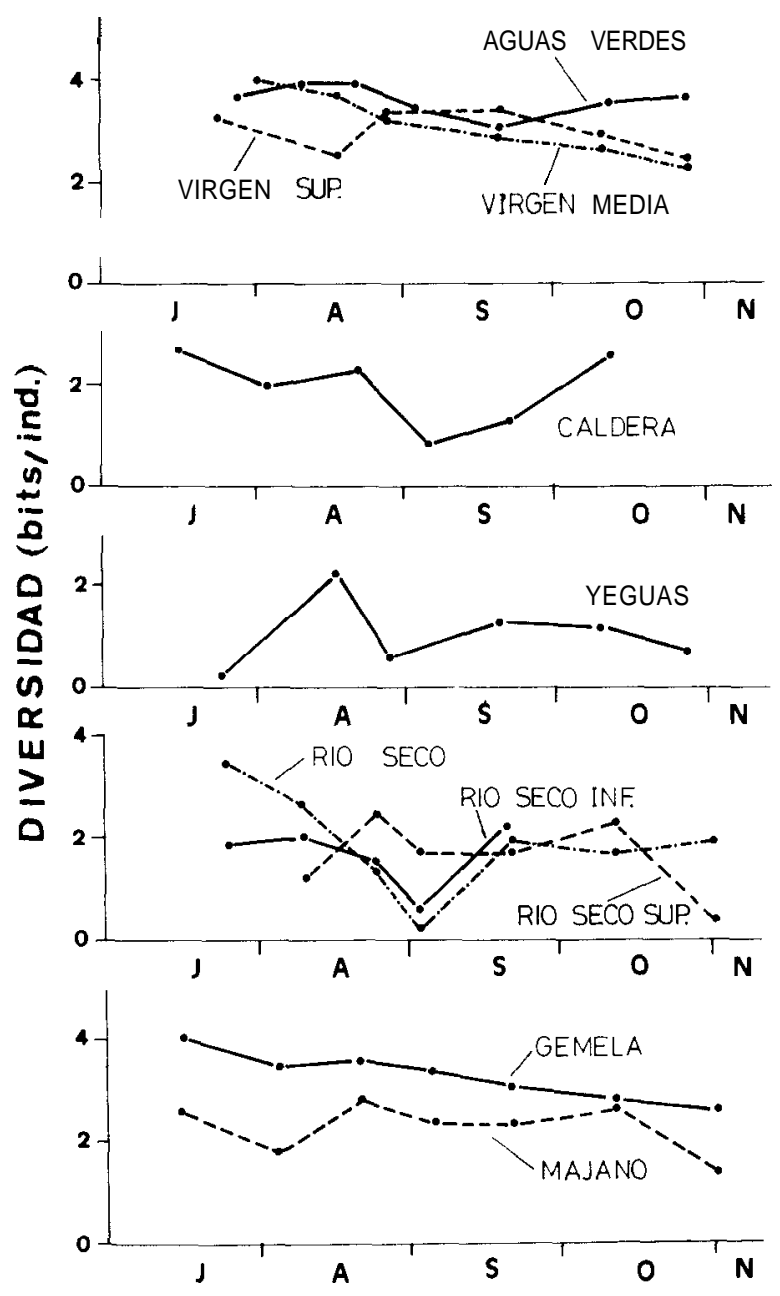

Figura 8.- Valores de diversidad (bitsiind) en el fitoplancton a lo largo del período libre de hielo.

Phytoplankton diversity values (bits/ind) during the ice-free period. riaciones que se observan suelen corresponder a cambios en la equitabilidad antes que en la riqueza de especies.

Este patrón general es claramente el que muestran las lagunas de Aguas Verdes, Virgen Media y Virgen Superior (fig. 8). En esta última, un desarrollo apreciable de la población de Oscillatoria agardhii es responsable del descenso en los valores del índice a mediados de agosto. La existencia de máximos poblacionales muy definidos en el tiempo determina, igualmente, las fluctuaciones de los valores de diversidad observados en las lagunas de La Caldera y Las Yeguas (fig. 8). Según Martínez (1975), en la primera de ellas, Cyanarcus sp. y Chromulina mikroplankton son las dos especies que dominan en la zona limnética. Nuestros resultados ponen de manifiesto que en las proximidades de la zona litoral tan sólo Cyanarcus sp. llega a ser dominante, con un desarrollo poblacional máximo hacia comienzos de septiembre, que da lugar al valor mínimo de diversidad medido.

En la laguna de Las Yeguas se han observado los valores más bajos del índice de diversidad. Su evolución en el tiempo muestra un mínimo destacable al final del deshielo, cuando la comunidad está dominada por Chlamydomonas sp. y, tras un breve período de uniformidad en la distribución de las abundancias específicas, los valores del índice vuelven a descender como consecuencia de la aparición, y dominancia, de Synedra rumpens.

Las lagunas del valle de Río Seco muestran un patrón similar en la evolución de la diversidad, con máximos particularmente notables en la laguna de Río Seco hacia finales del período de deshielo, y descensos progresivos hasta momentos en los que se sitúan las mayores densidades poblacionales de Korshikoviella gracilipes. En Río Seco Superior se detecta, además, un descenso brusco hacia final del período de estudio, que es debido tanto a una disminución en la riqueza específica, como a la dominancia que ejerce en estos momentos Cyclotella sp.

La laguna de Majano (fig. 8) presenta valores comprendidos entre 2 y 3 bitslindividuo; sólo a final de julio (dominancia de Staurastrum punctulatum) y a final de octubre (dominancia de Synechococcus maior) el índice de diversidad decrece considerablemente. En la laguna Gemela, donde 
la contribución parcial - e n número de individuos - de una especie nunca supera el $45 \%$ del total, se han medido valores elevados, comprendidos entre 2,6 y 3,9 bitsiindividuo.

c) Pigmentos fotosintetizadores

En la mayoría de las lagunas estudiadas, los valores promedio de la concentración de clorofila han estado comprendidos entre 0,5 y $2 \mu \mathrm{g} / \mathrm{l}$, y $\tan$ sólo las de Río Seco Inferior y Gemela han presentado máximos puntuales superiores a $5 \mu \mathrm{g} / 1$ (fig. 9); este rango de variación es semejante al que se cita para otros lagos de alta montaña (MARgalef et al., 1975; FerRari \& Villani, 1978; Balvay, 1978; Schanz, 1984; Duthie, 1979; KALFF et al., 1972). No parece existir un patrón definido en la evolución de estos valores en las distintas lagunas. Las del valle de Río Seco, así como las de Majano y Las Yeguas, muestran máximos más o menos definidos tras el deshielo, coincidiendo con el desarrollo de poblaciones de clorofíceas (Las Yeguas: Chlamydomonas sp. en una fase muy activa de crecimiento; Río Seco: Chlamydomonas cf. conifurmis) o de zigofíceas (Río Seco Inferior: Cylindrocystis brebissonii , Majano: Staurastrum hirsutum). En las lagunas de Río Seco Inferior y Río Seco destacan, además, máximos de clorofila de menor entidad hacia mediados del período de estudio, que corresponden al desarrollo de K. gracilipes en su fase ankiroide, principalmente.

El máximo poblacional de Synedra rumpens que se observa en la laguna de Las Yeguas a mediados de agosto no parece corresponder a los valores de clorofila. Esto estaría de acuerdo con las observaciones de Munawar \& Burns (1976), quienes no encuentran relación entre clorofila a y biomasa total en primavera, cuando las especies más abundantes de la comunidad pertenecen a diatomeas y criptomonadales, en el lago Eire.

El resto de las lagunas presentan los máximos valores de concentración de pigmentos hacia el final del período libre de hielo, que suele coincidir con el desarrollo de poblaciones de cianofíceas de diferentes tamaños. Este es el caso de las lagunas Gemela y Caldera. La primera presenta, en general, valores más altos de clorofila y, aunque se aprecian variaciones a lo largo de todo el período (que corresponden con el desarrollo de Chlorangiella pygmaea y Oocystis lacustris), la mayor con- centración se midió coincidiendo con una proliferación de Synechococcus maior, después de comenzar un nuevo período de helada en la laguna. En el caso de La Caldera, una laguna con valores muy bajos de clorofila, el máximo que se aprecia hacia finales de septiembre se corresponde, igualmente, con el desarrollo de Cyanarcus sp.
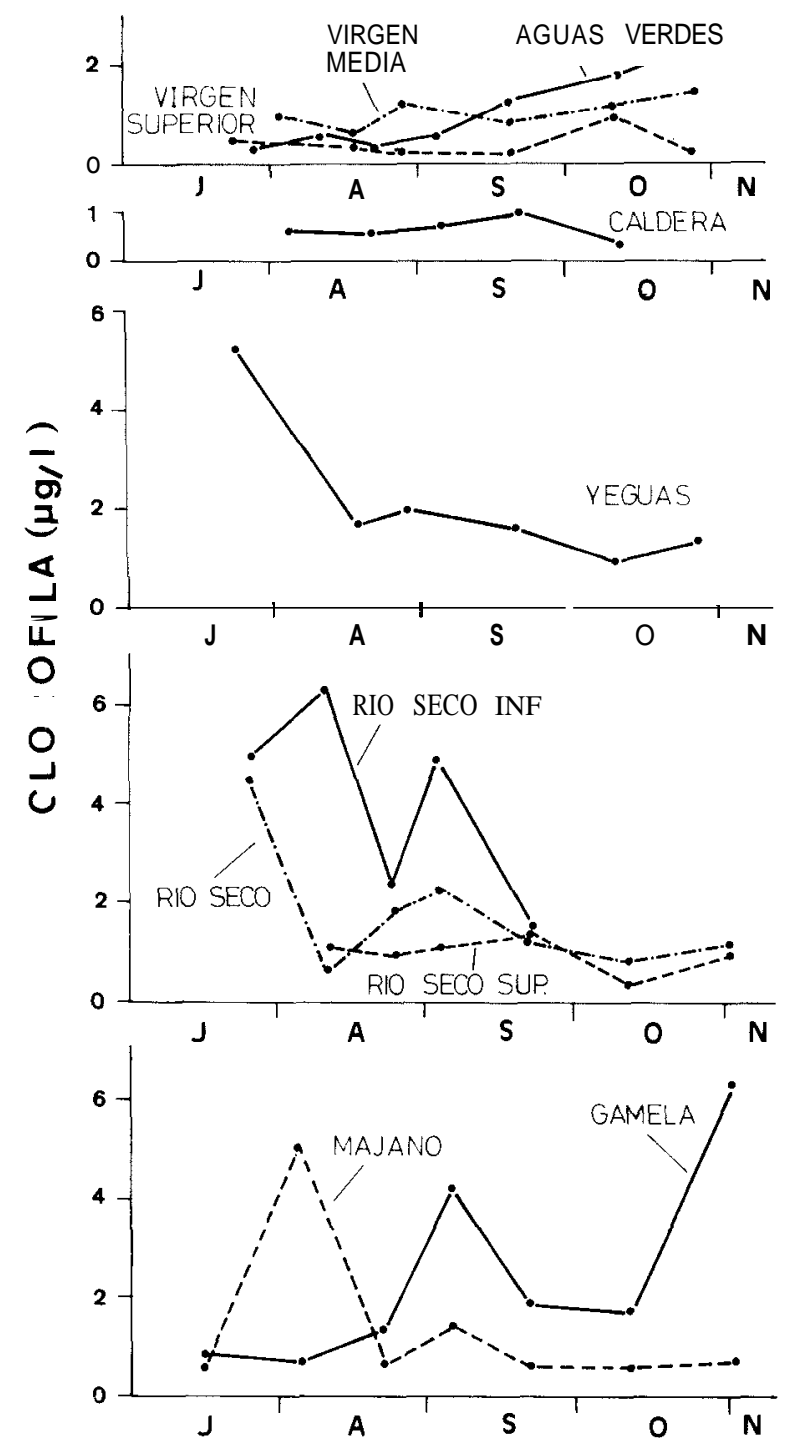

Figura 9.- Variación en la concentración de clorofila a $(\mu \mathrm{g} / \mathrm{l})$ en las lagunas estudiadas.

Seasonal variations in the chlorophyll a values $(\mu \mathrm{g} / 1)$ in the lakes studied. 
Las lagunas de Aguas Verdes, Virgen Media y Virgen Superior, particularmente las dos primeras, presentan máximos hacia el final del período de estudio, no atribuibles a una población en especial. Parece lógico pensar, en estos casos, en una influencia de los cinturones de vegetación litoral, entre los que se desarrollan importantes masas de algas filamentosas (sobre todo especies de los géneros Zygnema y Spirogyra), que desde comienzos de septiembre adquieren su máximo desarrollo.

\section{Bibliografía}

Anderson, R.S., 1970. Physical and chemical limnology of two mountain lakes in Banff National Park, Alberta. J. Fish. Res. Bd. Can. 27: 233-249.

American Public Health Association, 1965. Standard Methods for the Examination of Water and Wastewater, including Bottom Sediments and Sludge. 12th Ed. APHA. 769 págs.

BALVAY, G., 1978. Un lac oligotrophe de haute montagne: le Lac Cornu (Haute Savoie). Rev. Géogr. Alp. 66: 31-41.

Balvay, G. \& B. Blavoux, 1981. Le grand lac Jovet (Haute Savoie), milieu oligotrophe de haute montagne. Rev. Géogr. Alp. 69: 421-422.

Barica, G. \& F.A.J. ARmstrong, 1971. Contribution by snow to the nutrient of Ontario Lakes. Limnol. Oceanogr. 16: 891-899.

CAPBlancQ, J., 1972. Phytoplankton et productivité primaire de quelques lacs d'altitude dans les Pyrenées. Annls. Limnol. 8: 231-321.

CAPBlanCQ, J. \& H. LAville, 1968. Etude morphometrique et physico-chimique de neuf lacs du massif de Néouville (haute-Pyrenées). Annls. Limnol. 4: 275-324.

CARTER, J.R., 1970. Diatoms from Andorra. Beihefte zur Nova Hedwigia, Heft 31. Diatomaceae II. Gerloff, J., \& CHOLNOKY, B.J. Eds. 605-632

DUTHIE, H.C., 1979. Limnology of subartic Canadian lakes and some effects of impoundment. Arct. Alp. Res. 11: 145-158.

ELLSWORTH, P.M., 1983. Ecological seasonal cycles in a Colorado Mountain Pond. J. Fresh. Ecol. 2(3): 225-237.

Ferrari, I., C. Bellavere, L. Camurri \& M. Castellani, 1975. Limnologia física e chirnica e contenuti di clorofilla-a nel fitoplancton di un lago di montagna, il lago Sant Parmense. Riv. Biol. 14: 13-49.

FERRARI, I. \& M. VILLANI, 1978. Ricerche su fitoplancton e fitobentos in un lago di montagna, il Lago Santo Parmense. Gior. Bot. Ital. 112: 229-237.

GaUch, H.G., 1986. Multivariate Analysis in Community Ecology. Cambridge University Press. 298 págs.

Golterman, H.L., 1969. Methods for chemical analysis of fresh waters. IBP Handbook No. 8. Blackwell. Oxford and Edinburgh. 166 págs.
GonZÁlez Guerrero, P., 1927. Contribución al conocimiento ficológico del Pirineo español. Bol. R. Soc. Esp. Hist. Nat. 27: 343-346.

González Guerrero, P., 1965. Algas de la Sílice (Guadarrama). Annls. Inst. Bot. Cavanilles 23: 93-103.

Groterud, 0., 1972. Nutrients in ice. Some data from a high mountain lake. Verh. Internat. Verein Limnol. 18: 327-333.

Holm-Hansen, O. \& B. Riemann, 1978. Chlorophyll a determination: improvements in methodology. Oikos 30: 348-447.

Hutchinson, G.E., 1967. A treatise on Limnology. 2. Introduction to lake biology and the limnoplankton. John Wiley \& Sons. 1115 págs.

KalfF, J., H.E. Welch \& S.K. Holmgren, 1972. Pigment cycles in two high-artic Canadian lakes. Verh. Internat. Verein Limnol. 18: 250-256.

Kilham, S.S. \& P. KILHAM, 1984. The importance of resource supply rates in determining phytoplankton community structure. In: Trophic interactions within aquatic ecosystems (MEYERS, D.G. \& J.R. STRICKLER, eds.): 7-27. AAAS Selected Symposium 85.

LaRSON, G.L., 1973. A limnology study of a high mountain lake in Mount Rainier National Park, Washington State; USA. Arch. Hydrobiol. 72: 10-48.

LEwIS, W.M., 1978. Analysis of succession in a tropical phytoplankton comrnunity and a new rneasure of succession rate. Amer. Natur. 112: 401-414.

Malueg, K.W., J.R. Tilstra, D.W. Schults \& C.F. PoWERS, 1978. Limnological observation on an ultra-oligotrophic lake in Oregon, USA. Verh. Internat. Verein Limnol. 20: 292-302.

MARGalef, R., 1948. Flora, fauna y comunidades bióticas de las aguas dulces del Pirineo de la Cerdaña. Monogr. Est. Est. Pirenaicos 11: 1-226.

Margalef, R., 1949. La vida en los lagos de alta montaña de los Pirineos. Pirineos 11-12: 5-30.

Margalef, R., L. Campás, M.R. Miracle \& J.M. VilaseCA, 1975. Introducción al estudio de los lagos pirenaicos. Naturalia hispanica 4. Ministerio de Agricultura. ICONA. 47 págs.

MartíneZ, R., 1975. First report on the limnology of the alpine lake La Caldera in the Penibetic Mountains (Sierra Nevada Granada, Spain). Verh. Internat. Verein Limnol. 19: 1133-1139.

MartíneZ, R., 1977. Phytoplankton species, biomass, and diversity in lake La Caldera (Sierra Nevada, Granada, Spain). Acta Hydrobiol. 19: 95-107.

MARTínEZ, R., 1980. Seasonal variations of phytoplankton biomass and photosynthesis in the high-mountain lake La Caldera (Sierra Nevada, Spain). In: Developments in Hydrobiology. Vol. 3 (DokUlil, M., H. MeTZ \& D. JEWSON, eds.): 111-119. The Hague.

MARTínEZ, R., 1981. Nota sobre la migración vertical diaria de Rhodomonas minuta var. nannoplanktonica Skuja en la laguna de La Caldera. Bol. R. Soc. Esp. Hist. Nat. 78: 217-221.

Messikommer, E., 1942. Beitrag zur kenntnis der Algenflora und Algenvegetation des Hochgebirges um Davos. Beitrage zur geobotanischen Landesaufnahme der Schweiz 24. Hans Huber. Bern. 451 págs. 
Morales, R., L. Cruz-Pizarro \& P. Carrillo, 1986. Lagunas de alta montaña en Sierra Nevada: Algunas características físicas y químicas. Actas $\mathbf{1 1}$ Simposio sobre el Agua en Andalucía: 413-424.

Munawar, M. \& N.M. Burns, 1976. Relationships of phytoplankton biomass with soluble nutrients, primary production, and chlorophyll-a in lake Erie, 1970. J. Fish. Res. Bd Can. 33: 601-611.

MurPhy, J. \& J.P. RILEY, 1962. A modified single solution method for the determination of phosphate in natural waters. Analyt. Chim. Acta 27: 31-36.

Pechlaner, R., 1966. Die Finstertaler Seen (Kühtai, Osterreich). 1. Morphometrie, Hydrographie, Limnophysik und Limnochemie. Arch. Hydrobiol. 62: 165-230.

PeChlaner, R., 1967. Die Finstertaler Seen (Kühtai, Osterreich). II. Das Phytoplankton. Arch. Hydrobiol. 63: 145-193.

PenNaK, R.W., 1968. Field and experimental winter limnology of three Colorado mountain Lakes. Ecology 23: 141-161.

REYNOLDS, C.S., 1984. Phytoplancton periodicity: the interactions of form, function and environmental variability. Freshwat. Biol. 14: 111-142.

RoDIER, J., 1981. Análisis de las aguas naturales. Ed. Omega. Barcelona.

Round, F.E., 1981. The ecology of Algae. Cambridge University, Press Cambridge. 653 págs.

SánCHEZ-Castillo, P., 1984. Estudio ficológico de la vegetación reófila de Sierra Nevada (Granada). Limnetica 1: 136-140.

Sánchez-CaStlllo, P., 1987a. Un nuevo taxon del género Chromulina: C. nevadensis P.M. Sánchez sp nov. Cryptogamie. Algologie 8(4): 235-240.
SancheZ-Casthlo, P., 1987b. Ciclo biológico de Korshiko viella gracilipes (Lambert) Silva (Chlorococcales, Chlorophyta). Phycologia, 26(4): 496-500.

SÁncheZ-CAstillo, P., 1988. Algas de las lagunas de alta montaña de Sierra Nevada (Granada, España). Acta Botánica Malacitana 13: 21-52.

ScHANZ, F., 1984. Chemical and algological characteristics of five high mountain lakes near the Swiss National Park. Verh. Internat. Verein Limnol. 22: 1066-1070.

Shannon, C.E. \& W. WEAVER, 1963. The mathematical theory of comunication. Univ. Illinois Press. Urbana.

SOMmer, U., 1981. The role of $r$ - and K-selection in the succession of phytoplankton in lake Constance. Acta Oecologica 2: 327-342.

Talling, J.F. \& D. Driver, 1963. Some problems in the estimation of chlorophyll-a in phytoplankton. Proc. Conference on Primary Productivity measurement, Marine and Freshwater, Hawaii 1961. U.S. Atomic Energy Comm. TID-7633: 142-146.

Thomasson, K., 1956. Reflections on Artic and Alpine lakes. Oikos 7: 117-143.

Tilman, D., S.S. Kilham \& P. Kilham, 1982. Phytoplancton comunity ecology: The role of limiting nutrients. Ann. Rev. Ecol. Syst. 13: 349-372

Tilman, G.D., S.S. Kilmam \& P. Kilham, 1984. A reply to sell, Carney and Fahnenstie. Ecology 65: 328-332.

Turnowsky, F., 1961. Der Wolayersee in der Karnischen hauptkette. Carinthia 2(71): 102-126.

UTERMOHL, H., 1958. Zur Vervollkommung der quantitativen Phytoplanktonmethodik. Mitt. int. Ver. Limnol. 9: $1-38$.

VilaseCA, J.M., 1978. Fitoplancton de los lagos pirenaicos. Tesis de licenciatura. Univ. Barcelona.

Wetzel, R.G., 1981. Limnología. Ed. Omega. Barcelona. 679 págs. 